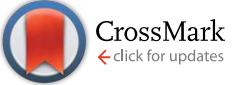

Cite this: RSC Adv., 2017, 7, 17763

Received 11th January 2017

Accepted 10th February 2017

DOI: 10.1039/c7ra00463j

rsc.li/rsc-advances

\section{Low temperature hydrothermal synthesis of battery grade lithium iron phosphate $\uparrow$}

\author{
Peter Benedek, Nils Wenzler, Maksym Yarema and Vanessa C. Wood*
}

Lithium ion transport through the cathode material $\mathrm{LiFePO}_{4}$ (LFP) occurs predominately along onedimensional channels in the [010] direction. This drives interest in hydrothermal syntheses, which enable control over particle size and aspect ratio. However, typical hydrothermal syntheses are performed at high pressures and are energy intensive compared to solid-state reactions, making them less practical for commercial use. Here, we show that the use of high precursor concentrations enables us to achieve highly crystalline material at record low-temperatures via a hydrothermal route. We produce LFP platelets with thin [010] dimensions and low antisite defect concentrations that exhibit specific discharge capacities of $150 \mathrm{~mA} \mathrm{~h} \mathrm{~g}{ }^{-1}$, comparable to material produced with higher temperature syntheses. An energy consumption analysis indicates that the energy required for our synthesis is $30 \%$ less than for typical hydrothermal syntheses and is comparable to solid-state reactions used today, highlighting the potential for low temperature hydrothermal synthesis routes in commercial battery material production.
Lithium iron(II) phosphate (LFP) is a commercially-used lithium ion battery (LIB) cathode material that offers some advantages over other cathode materials due to the fact that it does not contain cobalt, and that it has a flat voltage profile and a high rate capability. ${ }^{1}$ It is commercially produced in a solid-state synthesis route; however, this well-established preparation offers less morphology and composition control than wet chemistry approaches. ${ }^{2}$ A large number of solvothermal syntheses have emerged for LFP, ${ }^{3}$ especially using water as the solvent (i.e., hydrothermal syntheses). ${ }^{3-12}$ Typically, these hydrothermal syntheses are performed with a precursor concentration in the range of $0.1-0.3 \mathrm{M}$, at temperatures between $150{ }^{\circ} \mathrm{C}$ and $200{ }^{\circ} \mathrm{C}$, and for a time in the order of ten hours. ${ }^{5,7-9}$ Rather high process temperatures (150$200{ }^{\circ} \mathrm{C}$ ) pose two issues towards commercialization: (i) the energy consumption for hydrothermal production of LFP is significantly higher than for the solid-state LFP synthesis and (ii) the vapor pressure of water can reach more than $10 \mathrm{bar}$, requiring a special reactor design. ${ }^{13}$ A commercially viable LFP hydrothermal synthesis will therefore require reduction of the reaction temperature.

To find the parameters at which the specific energy consumption of a hydrothermal synthesis process could compete with the solid state one, we develop a model to estimate the energy required for the hydrothermal-based synthesis

Laboratory of Nanoelectronics, Department of Information Technology and Electrical Engineering, ETH Zurich, CH-8092 Zurich, Switzerland. E-mail: vwood@ethz.ch

$\dagger$ Electronic supplementary information (ESI) available: Energy consumption analysis, description of the sampling tool, X-ray diffraction data and Rietveld refinements, characterization of carbon coatings, additional electrochemical data, and particle morphology characterization. See DOI: 10.1039/c7ra00463j of LFP from mine to ready-to-use cathode material. Extending upon existing literature, ${ }^{\mathbf{1 3 - 1 8}}$ we consider the impact of the key parameters such as synthesis temperature, precursor concentration, and reaction time on the specific energy consumption. Details of the model are given in the ESI, $\uparrow$ and the dependence of the specific energy on reaction temperature and precursor concentration is shown in Fig. 1. The energy consumption of the hydrothermal synthesis is largely related to the large heat capacity of water, which makes the heating of the reactor

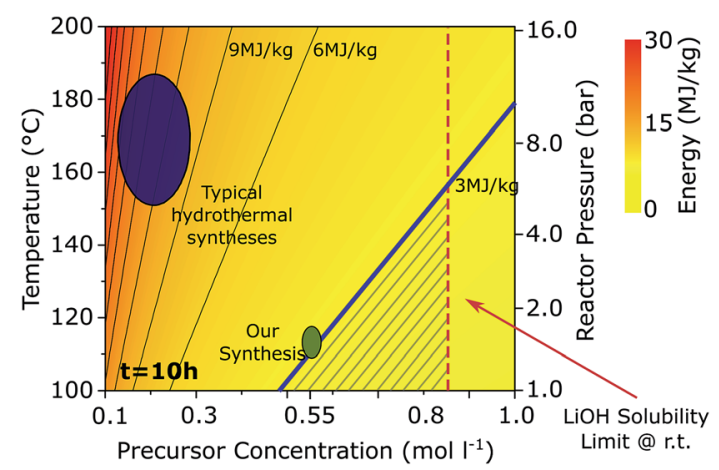

Fig. 1 Specific energy consumption of the LFP synthesis step as a function of hydrothermal reaction temperature and total precursor concentration. The blue shaded region indicates the energy consumption of typical hydrothermal syntheses, the synthesis reported here is indicated green. The iso-energy line corresponding to 3

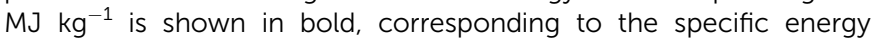
consumption of a solid-state synthesis. The shaded triangular region below this line represents a temperature and precursor concentration window where a hydrothermal synthesis consumes less energy than a solid-state synthesis. 
(not the time held at a specific temperature) energy intensive. Our model shows that the reaction temperature as well as the precursor concentration, $C_{\text {tot }}$ play a role in energy usage. The larger $C_{\text {tot }}$, the smaller the specific energy consumption because, at lower concentrations, a smaller mass of LFP is created for the same heated volume of water. For temperatures of $150{ }^{\circ} \mathrm{C}$, a hydrothermal synthesis can only compete with the 3 $\mathrm{MJ} \mathrm{kg^{-1 }}$ energy consumption of a solid-state synthesis if the precursor concentration were at the solubility limit of $\mathrm{LiOH}$ in water. With decreasing the reactor temperature, the dependence of energy consumption on concentration weakens, allowing a larger range of possible precursor concentrations.

Based on this analysis, we aim to develop a synthesis at $115{ }^{\circ} \mathrm{C}$ that still yields battery grade material. At this temperature, the specific energy consumption can be lowered to $3 \mathrm{MJ}$ $\mathrm{kg}^{-1}$ using a feasible precursor concentration (i.e., one below the solubility limit) and the vapor pressure in the reactor will be about 1.5 bar, presenting convenient operating conditions, similar to a pressure cooker.

The hydrothermal synthesis of high-quality LFP at low temperatures is a challenging chemistry task..$^{4-6}$ Low temperature syntheses result in slow growth kinetics and produce LFP with poor crystallinity and a large number of antisite defects (i.e., Fe atoms populating the Li positions). These antisite defects block the [010] Li transport channels in the LFP structure and ultimately decrease specific charge capacities. ${ }^{19-22}$ Moreover, the slow growth kinetics lead to a low number of nuclei, leading to thicker LFP particles and thus longer [010] Li channel lengths such that there is a higher probability of antisite defects blocking them. ${ }^{23}$

In this letter, we present a study of low-temperature hydrothermal synthesis of LFP platelets. In particular, we optimize the precursor concentration and reaction time in order to achieve battery-grade LFP material. We then perform the carbon coating of LFP platelets and show electrochemical performance on par with that synthesized at higher temperatures with specific discharge capacities of up to $150 \mathrm{~mA} \mathrm{~h} \mathrm{~g}{ }^{-1}$ at $\mathrm{C} / 5$ and $120 \mathrm{~mA} \mathrm{~h} \mathrm{~g}^{-1}$ at $1 \mathrm{C}$ rate.

Scheme 1 shows our low temperature synthesis approach. As detailed in the Experimental methods, we use typical precursors for hydrothermal synthesis of LFP: $\mathrm{LiOH}, \mathrm{H}_{3} \mathrm{PO}_{4}$, and $\mathrm{FeSO}_{4}$ $\cdot 7 \mathrm{H}_{2} \mathrm{O}{ }^{4-11}$ We also use ethylene glycol (EG) as an additive, ${ }^{5,7}$ which is known to decrease the solubility of the precursors, increase the number of nucleation sites, and thereby achieve smaller particle dimensions. ${ }^{7}$ Here, to ensure that the role of the EG is primarily in coordination of the LFP surfaces and to be close to a possible commercial application, we work with small additive concentrations and select a fixed molar ratio of $[\mathrm{LiOH}]:\left[\mathrm{FeSO}_{4} \cdot 7 \mathrm{H}_{2}\right.$ $\mathrm{O}]:\left[\mathrm{H}_{3} \mathrm{PO}_{4}\right]:[\mathrm{EG}]=3: 1: 1: 0.1$ for all syntheses.

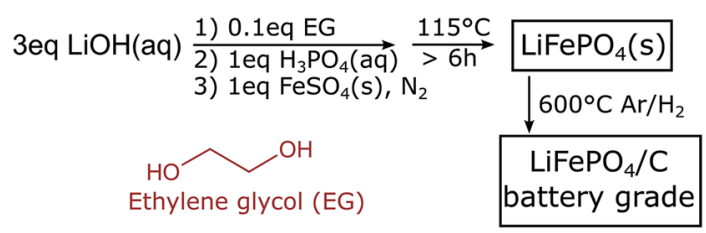

Scheme 1 Preparation procedure of battery grade $\mathrm{LiFePO}_{4}$.
To assess our ability to control material quality as well as particle shape and size with our low temperature hydrothermal synthesis, we systematically look at the effect of (1) precursor concentration and (2) reaction time. Since density function theory (DFT) calculations suggest that the symmetric stretching band of the $\mathrm{PO}_{4}$ group is redshifted when the antisite defect concentration is increased, ${ }^{5}$ we use Fourier transform infrared (FTIR) spectroscopy to assess material quality. Scanning electron microscopy (SEM) enables us to obtain approximate the particle morphology.

Even though it is generally accepted that the total precursor concentration influences the supersaturation ${ }^{24}$ and therefore the reaction kinetics, crystallinity, and particle size, it has not been extensively studied in hydrothermal syntheses of LFP. ${ }^{11}$ We investigate measurements performed on LFP samples after 16 hours of reaction. The FTIR spectra of the $\mathrm{PO}_{4}$ stretching modes of each sample at different concentrations are shown in Fig. 2a. Increasing the precursor concentration, $C_{\text {tot }}$, results in LFP particles that show sharper spectral features and a shift of the $\mathrm{PO}_{4}$ stretching band towards lower wavenumbers, both indicating smaller defect concentration (Fig. 2b). These FTIR results are consistent with Rietveld refinements on X-Ray diffractograms (Fig. 2S $\dagger$ ), from which we find a decrease of antisite defects with increased $C_{\text {tot }}$ (Fig. 3S $\dagger$ ). The decrease in antisite defect with increasing precursor concentration can be explained by faster reaction kinetics at higher concentrations, whereby the LFP crystals form faster and have more time within the set reaction time of 16 hours to recrystallize. This is supported by SEM images (Fig. 2c) showing that, at low $C_{\text {tot }}$, the particles are not all evolved, while uniform platelets are formed for increasing $C_{\text {tot }}$.

Increasing the precursor concentration also affects the particle morphology. With increasing $C_{\text {tot }}$, the average particle thickness decreases from $220 \mathrm{~nm}$ to $150 \mathrm{~nm}$ (Fig. 2d) while the platelet diameter remains approximately constant around 1.6 $\mu \mathrm{m}$ (Fig. 2e). These results can be explained by DFT calculations $^{21}$ and previous experimental results, ${ }^{5,7}$ which suggest that both water and EG cap the (010) facet, inhibiting growth in the [010] direction.

To investigate the optimal reaction time for a low temperature hydrothermal synthesis, we construct a sampling reactor that enables us to remove material at different times during the reaction (Fig. $4 \mathrm{~S}_{\dagger} \dagger$ ). We consider a reaction with a precursor concentration $C_{\text {tot }}=0.55$ M. As shown in Fig. 3a, with increasing reaction time, the average particle thickness tends to increase slightly and the distribution of thicknesses broadens (i.e., $130 \mathrm{~nm} \pm 50 \mathrm{~nm}$ at 3 hours to $150 \mathrm{~nm} \pm 70 \mathrm{~nm}$ at 72 hours), reflecting the Ostwald ripening process. ${ }^{25}$ At the same time, defect concentration (as quantified by the peak position of the symmetric $\mathrm{PO}_{4}$ stretching band) decreases with longer reaction times (Fig. $3 \mathrm{~b}$ ) due to recrystallization of LFP. This poses a trade-off, because for the highest charge capacity both thin platelet morphology LFP particles (i.e., short [010] Li channels) and low antisite defect concentrations are required. We thus expect a reaction time in the middle of those tested (e.g., 48 hours) to exhibit the best electrochemical performance. 

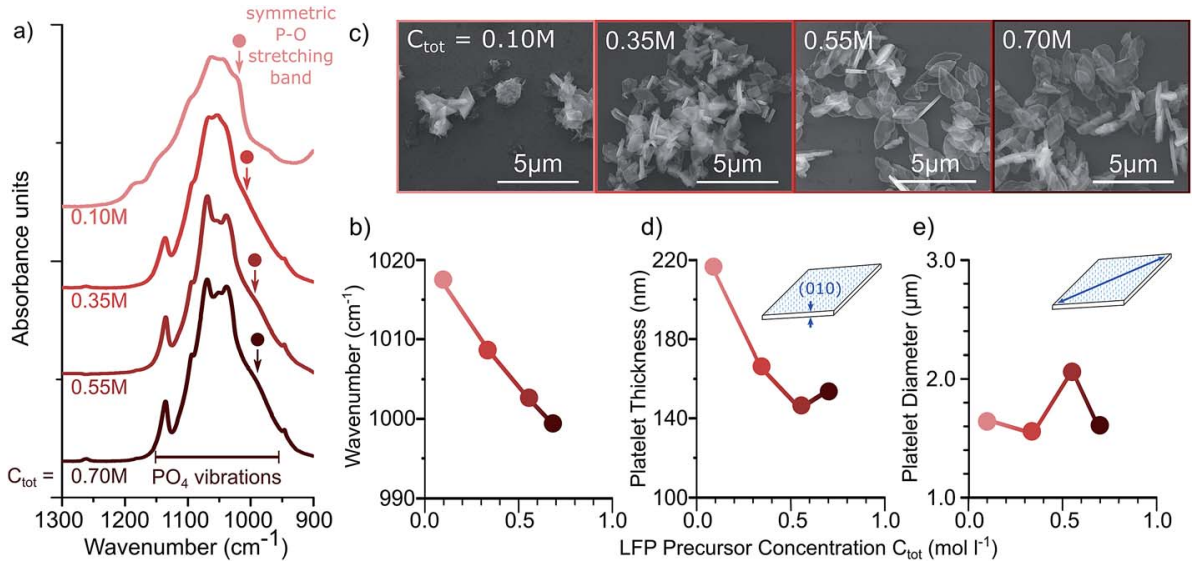

Fig. 2 Dependence of defect concentration and particle morphology as a function of molar concentration of precursors, $C_{\text {tot }}$ in water. Precursors are fixed in a ratio of $[\mathrm{LiOH}]$ : $\left[\mathrm{FeSO}_{4} \cdot 7 \mathrm{H}_{2} \mathrm{O}\right]:\left[\mathrm{H}_{3} \mathrm{PO}_{4}\right]$ : [EG] $=3: 1: 1: 0.1$. (a) FTIR spectra and (b) position of the symmetric P-O stretching band peak for samples synthesized at different precursor concentrations. (c) SEM images, (d) mean particle thickness, and (e) mean particle diameter are also shown for different precursor concentrations.

To test the electrochemical performance of our newly obtained material, we prepare the LFP particles for electrochemical cycling by carbon coating them. The LFP particles are mixed with D-glucose, pressed into pellets, and annealed at $600{ }^{\circ} \mathrm{C}$ resulting in LFP particles with a coating of $3 \mathrm{wt} \%$ of carbon. XRD, FTIR, SEM, and transmission electron microscope (TEM) measurements suggest that the carbon coating step removes the solvent incorporated in the crystal and provides a conductive coating but does not affect the particle crystal structure or the antisite defect concentration. As described in the methods, electrodes are made from different particle batches and cycled $v s$. lithium in a half-cell configuration.

Fig. $3 \mathrm{c}$ shows the electrochemical voltage $v s$. capacity curves for $\mathrm{C} / 10$ (dis)charge for three LFP batches prepared with the
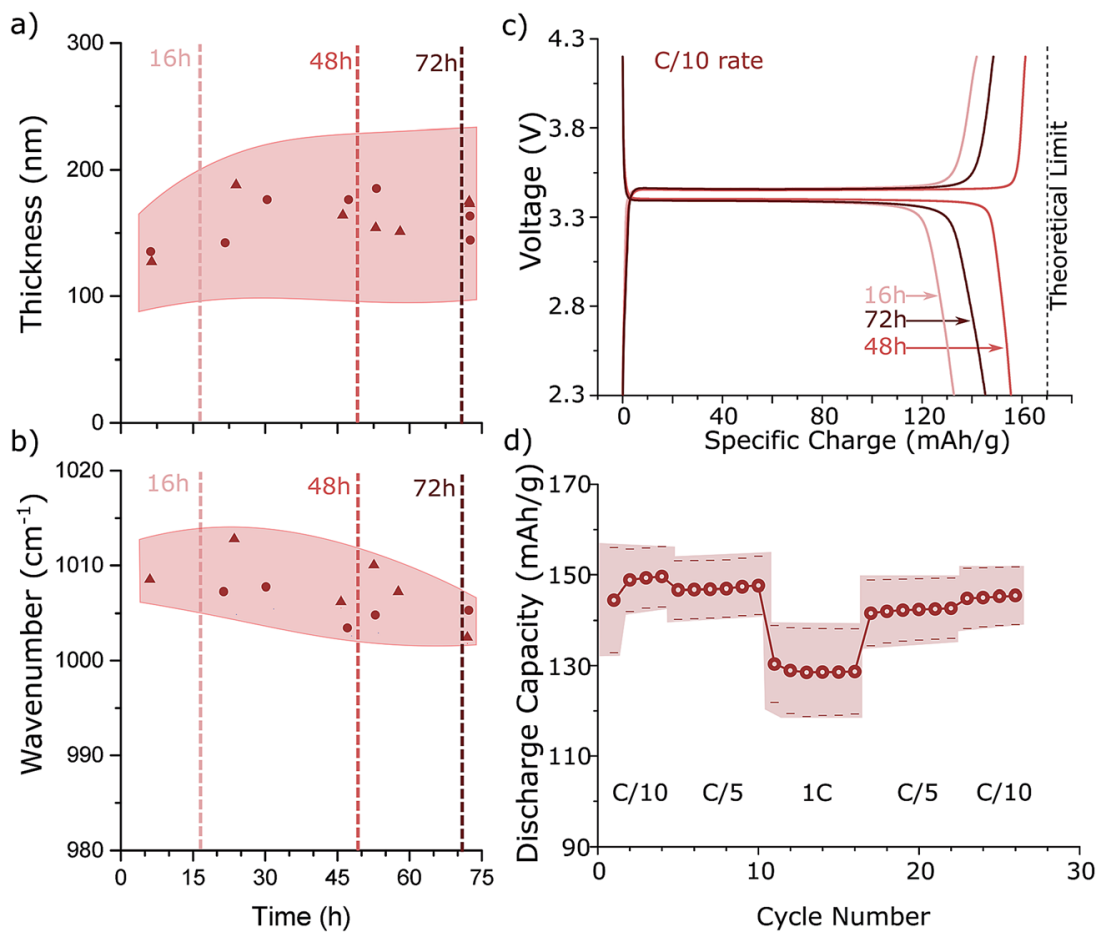

Fig. 3 Two different batches (circles, triangles) of LFP platelets synthesized under the same conditions are sampled as a function of reaction time and their thickness (a) and the peak position of the symmetric $\mathrm{P}-\mathrm{O}$ stretching mode (b) is plotted. Shading indicates the standard deviation of the measurements. (c) Polarization curves at C/10 rate of LFP samples prepared with different reaction times. (d) Specific discharge capacity at different $C$ rates $\left(1 \mathrm{C}=0.17 \mathrm{~A} \mathrm{~g}^{-1}\right)$ of LFP synthesized at $115^{\circ} \mathrm{C}$ at a precursor concentration of $C_{\text {tot }}=0.55 \mathrm{M}$ and a reaction time of $48 \mathrm{~h}$. Shading indicates the error bars on the measurements, which are determined by measuring 5 cells containing different electrodes prepared from two different batches of material. 
same precursor concentration of $C_{\text {tot }}=0.55 \mathrm{M}$ and reaction times of 16, 48, and 72 hours. All three samples exhibit a flat voltage plateau and low polarization at a $\mathrm{C} / 10$ rate. Our parameter sweep indicated that the best electrochemical performance should result from particles synthesized for $\sim 48$ hours, and indeed this sample performs best with a specific

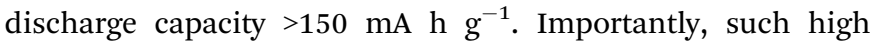
discharge capacity of LFP material is already comparable to values obtained by high temperature hydrothermal syntheses and improves upon previous reports for low temperature hydrothermal LFP synthesis. ${ }^{4,5}$ Furthermore, our LFP samples show only very small capacity fading after 50 charge/discharge cycles (Fig. 6S $\dagger$ ). Using galvanostatic cycling, the specific discharge capacity at different $\mathrm{C}$ rates $\left(1 \mathrm{C}=0.17 \mathrm{~A} \mathrm{~g}^{-1}\right)$ is determined from the LFP particles synthesized for 48 hours. Fig. 3d shows the average discharge capacity and standard deviation of sample electrodes prepared in different batches. The specific charge capacity drops to $130 \mathrm{~mA} \mathrm{~h} \mathrm{~g}{ }^{-1}$, when the cycling rate is increased to $1 \mathrm{C}$, which is comparable to high temperature hydrothermal LFP syntheses. ${ }^{4-11}$

Table 1 summarizes the specific energy consumption of our proposed hydrothermal synthesis, a high temperature hydrothermal approach proposed by Chen et al. ${ }^{\mathbf{6}}$ and a solid state synthesis. This comparison includes the carbon-coating step in our hydrothermal synthesis for which we assume an extra energy consumption of $2 \mathrm{MJ} \mathrm{kg}^{-1}$. By increasing the concentration and decreasing reaction time, our synthesis saves more than $30 \%$ compared to high temperature hydrothermal syntheses, but still consumes more energy than solid state syntheses. However, an excess of lithium is used in the hydrothermal process, which leads to the formation of $\mathrm{Li}_{2} \mathrm{SO}_{4}$. If this co-product was recycled, the precursor energy consumption would decrease by up to $50 \%$ eventually making the synthesis beneficial from an energy perspective. Of course, energy consumption during synthesis is only a small part of the costs of the overall material production. For instance, the aforementioned temperature dependent pressure in the reactor has a high impact on investment cost due to increased safety requirements. However, while increased reaction time has only a small effect on energy consumption (heating up to and not holding the temperature is the energy intensive step as highlighted in Fig. 7S $\dagger$ ), it does impact throughput. Therefore, a multi-parameter optimization of temperature, reaction time, and the process (e.g. using flow reactor set-up) would be necessary for commercial introduction of a hydrothermal process.

In conclusion, we synthesized LFP platelet particles in a hydrothermal synthesis with a low reaction temperature of

Table 1 Overview of the specific energy consumption of LFP prepared by different synthetic approaches

\begin{tabular}{llll}
\hline & $\begin{array}{l}\text { Solid state } \\
\text { synthesis }\end{array}$ & $\begin{array}{l}\text { High temp. } \\
\text { hydrothermal }\end{array}$ & $\begin{array}{l}\text { Our } \\
\text { approach }\end{array}$ \\
\hline $\begin{array}{l}\text { Synthesis energy } \\
\text { Precursors energy }\end{array}$ & $\begin{array}{l}3 \mathrm{MJ} \mathrm{kg}^{-1} \\
22 \mathrm{MJ} \mathrm{kg}^{-1}\end{array}$ & $\begin{array}{l}19 \mathrm{MJ} \mathrm{kg}^{-1} \\
26 \mathrm{MJ} \mathrm{kg}^{-1}\end{array}$ & $\begin{array}{l}5 \mathrm{MJ} \mathrm{kg}^{-1} \\
26 \mathrm{MJ} \mathrm{kg}^{-1}\end{array}$ \\
$\begin{array}{l}\text { Reactor pressure } \\
\text { Total }\end{array}$ & $\begin{array}{l}10 \mathrm{bar} \\
25 \mathrm{MJ} \mathrm{kg}^{-1}\end{array}$ & $45 \mathrm{MJ} \mathrm{kg}^{-1}$ & $\begin{array}{l}1.6 \mathrm{bar}^{-1} \\
\text { MJ kg }\end{array}$
\end{tabular}

$115^{\circ} \mathrm{C}$. Increased precursor concentration together with small amounts of EG as an additive lead to highly crystalline particles with a platelet-shaped morphology having small dimensions along [010]. Using a sampling setup, we found the counteracting effects of recrystallization and Ostwald ripening lead to an optimal reaction time in the range of 48 hours. Our analysis shows that reduction of reaction temperature and increase of precursor concentration can bring the energy consumption of a hydrothermal synthesis in line with that of a solid state approach, while enabling particle size and shape control not available with solid state approaches. This work highlights the open potential for optimization of hydrothermal processes at lower temperatures and higher concentrations.

\section{Experimental methods}

In a typical synthesis of LFP, a $20 \mathrm{~mL}$ solution of LiOH (Sigma) and the ethylene glycol (Sigma) is transferred into a $50 \mathrm{~mL}$ stainless steel reactor. While stirring, $20 \mathrm{~mL}$ of $\mathrm{H}_{3} \mathrm{PO}_{4}$ solution is added dropwise. A white precipitate of $\mathrm{Li}_{3} \mathrm{PO}_{4}$ forms. Ground $\mathrm{FeSO}_{4} \cdot 7 \mathrm{H}_{2} \mathrm{O}$ (Sigma) powder is quickly added to the dispersion and the reactor is purged with $\mathrm{N}_{2}$ for $10 \mathrm{~min}$. The final molar ratio $[\mathrm{LiOH}]:\left[\mathrm{FeSO}_{4} \cdot 7 \mathrm{H}_{2} \mathrm{O}\right]:\left[\mathrm{H}_{3} \mathrm{PO}_{4}\right]:[\mathrm{EG}]$ in the solution is kept $1: 1: 3: 0.1$. After purging, the reactor is heated with a heating mantle. When the synthesis is finished, the resulting off-white powder is washed in $\mathrm{H}_{2} \mathrm{O}$ and ethanol and dried at $80{ }^{\circ} \mathrm{C}$. To coat the LFP particles with $3 \mathrm{wt} \%$ of carbon, glucose is mixed thoroughly with the LFP particles. The mixture is pressed in a pellet and then heated at $600{ }^{\circ} \mathrm{C}$ for $6 \mathrm{~h}$ in $\mathrm{Ar} / 3 \mathrm{wt} \% \mathrm{H}_{2}$ stream. The resulting powder is black.

To determine the particle dimensions, scanning electron microscopy (SEM) images in secondary electron mode are taken using a Hitachi SU-8200. The dimensions of 100-200 particles are measured using ImageJ, see Fig. $8 \mathrm{~S} \dagger$ for details. FTIR spectra are obtained on an attenuated total reflection (ATR) setup with Ge single crystal on a Bruker Vertex 70 Fourier transformed infrared spectrometer. The observed symmetric $\mathrm{PO}_{4}$ stretching band is fit with a Gaussian function. X-ray powder diffraction spectra are measured in a $2 \theta$ range between $15^{\circ}$ and $85^{\circ}$ using a Rigaku Smartlab diffractometer. Rietveld refinements are performed with the Maud software. ${ }^{26}$ To analyze the Carbon coated samples, transmission electron micrographs are taken with a FEI F30 microscope.

To test LFP electrodes, $70 \mathrm{wt} \%$ of active material, $20 \mathrm{wt} \%$ of Super C64 carbon black (Timcal), and $10 \mathrm{wt} \%$ of Kynar HSV900 polyvinylidene fluoride binder are dispersed in $\mathrm{N}$-methyl-2pyrrolidone (NMP, Sigma). Resulting slurries are blade coated on an aluminum sheet and dried at $120{ }^{\circ} \mathrm{C}$ under vacuum for $8 \mathrm{~h}$. Half cells were prepared under argon atmosphere, using glass fiber separator soaked with $500 \mu \mathrm{l}$ of the electrolyte, a $1 \mathrm{M}$ solution of $\mathrm{LiPF}_{6}$ in 1:1 ethylene carbonate and dimethyl carbonate (BASF), between the LFP cathode and lithium metal reference. The cycling measurements are performed at room temperature on a Biologic VMP3 potentiostat. The reported values (data points) and errors (shaded regions) in Fig. 3 come from the average and standard deviation of measurements on 
different electrodes prepared with materials from different synthesis batches, but with the same reaction conditions.

\section{Acknowledgements}

The authors acknowledge funding from the European Research Council (ERC), the Swiss National Science Foundation (SNSF) precoR program. TEM measurements were performed at the Scientific Center for Optical and Electron Microscopy of the Swiss Federal Institute of Technology.

\section{References}

1 B. Kang and G. Ceder, Battery materials for ultrafast charging and discharging, Nature, 2009, 458, 190-193.

2 M. Yoshimura and K. Byrappa, Hydrothermal processing of materials: past, present and future, J. Mater. Sci., 2008, 43, 2085-2103.

3 C. Masquelier and L. Croguennec, Polyanionic (phosphates, silicates, sulfates) frameworks as electrode materials for rechargeable $\mathrm{Li}$ (or $\mathrm{Na}$ ) batteries, Chem. Rev., 2013, 113, 6552-6591.

4 O. Xiuqin, P. Lin, G. Haichen, W. Yichen and L. Jianwei, Temperature-dependent crystallinity and morphology of $\mathrm{LiFePO}_{4}$ prepared by hydrothermal synthesis, J. Mater. Chem., 2012, 22, 9064-9068.

5 X. Qin, J. Wang, J. Xie, F. Li, L. Wen and X. Wang, Hydrothermally synthesized $\mathrm{LiFePO}_{4}$ crystals with enhanced electrochemical properties: simultaneous suppression of crystal growth along [010] and antisite defect formation, Phys. Chem. Chem. Phys., 2012, 14, 2669-2677.

6 J. Chen, M. J. Vacchio, S. Wang, N. Chernova, P. Y. Zavalij and M. S. Whittingham, The hydrothermal synthesis and characterization of olivines and related compounds for electrochemical applications, Solid State Ionics, 2008, 178, 1676-1693.

7 S. Yang, X. Zhou, J. Zhang and Z. Liu, Morphology-controlled solvothermal synthesis of $\mathrm{LiFePO}_{4}$ as a cathode material for lithium-ion batteries, J. Mater. Chem., 2010, 20, 8086-8091.

8 Y. Li, J. N. Weker, W. E. Gent, D. N. Mueller, J. Lim, D. A. Cogswell, T. Tyliszczak and W. C. Chueh, Dichotomy in the Lithiation Pathway of Ellipsoidal and Platelet $\mathrm{LiFePO}_{4}$ Particles Revealed through Nanoscale Operando State-of-Charge Imaging, Adv. Funct. Mater., 2015, 25, 36773687.

9 Z. Cabán-Huertas, O. Ayyad, D. P. Dubal and P. GómezRomero, Aqueous synthesis of $\mathrm{LiFePO}_{4}$ with Fractal Granularity, Sci. Rep., 2016, 6, 27024.

10 Z. Lu, H. Chen, R. Robert, B. Y. X. Zhu, J. Deng, L. Wu, C. Y. Chung and C. P. Grey, Citric Acid- and AmmoniumMediated Morphological Transformations of Olivine $\mathrm{LiFePO}_{4}$ Particles, Chem. Mater., 2011, 23, 2848-2859.

11 B. Ellis, W. H. Kan, W. R. M. Makahnouk and L. F. Nazar, Synthesis of nanocrystals and morphology control of hydrothermally prepared $\mathrm{LiFePO}_{4}$, J. Mater. Chem., 2007, 17, 3248-3254.

12 K. Dokko, S. Koizumi, H. Nakano and K. Kanamura, Particle morphology, crystal orientation, and electrochemical reactivity of $\mathrm{LiFePO}_{4}$ synthesized by the hydrothermal method at 443 K, J. Mater. Chem., 2007, 17, 4803-4810.

13 J. B. Dunn, L. Gaines, J. C. Kelly, C. James and K. G. Gallagher, The significance of Li-ion batteries in electric vehicle life-cycle energy and emissions and recycling's role in its reduction, Energy Environ. Sci., 2015, 8, 158-168.

14 G. Majeau-Bettez, T. R. Hawkins and A. H. Strømman, Life Cycle Environmental Assessment of Lithium-Ion and Nickel Metal Hydride Batteries for Plug-In Hybrid and Battery Electric Vehicles, Environ. Sci. Technol., 2011, 45, 4548-4554.

15 M. C. Johnson, I. Palou-Rivera and E. D. Frank, Energy consumption during the manufacture of nutrients for algae cultivation, Algal Res., 2013, 2, 426-436.

16 C.-T. Liu and T. L. William Jr, Vapor pressure of deuterated water from 106 to $300 \mathrm{deg}, J$. Chem. Eng. Data, 1970, 15, 510513.

17 D. R. Stull, Vapor Pressure of Pure Substances. Organic and Inorganic Compounds, Ind. Eng. Chem., 1947, 39, 517-540.

18 CRC Handbook of Chemistry and Physics, W. M. Haynes, ed., CRC Press/Taylor \& Francis, Boca Raton, FL, 97th edn (Internet Version), 2017.

19 S.-i. Nishimura, G. Kobayashi, K. Ohoyama, R. Kanno, M. Yashima and A. Yamada, Experimental visualization of lithium diffusion in LixFePO $_{4}$, Nat. Mater., 2008, 7, 707-711.

20 L. Wang, F. Zhou, Y. Meng and G. Ceder, First-principles study of surface properties of $\mathrm{LiFePO}_{4}$ : surface energy, structure, Wulff shape, and surface redox potential, Phys. Rev. B: Condens. Matter Mater. Phys., 2007, 76, 16543.

21 C. A. J. Fisher and M. S. Islam, Surface structures and crystal morphologies of $\mathrm{LiFePO}_{4}$ : relevance to electrochemical behaviour, J. Mater. Chem., 2008, 18, 1209-1215.

22 S. Chung, S. Choi, T. Yamamoto and Y. Ikuhara, AtomicScale Visualization of Antisite Defects in $\mathrm{LiFePO}_{4}$, Phys. Rev. Lett., 2008, 100, 125502.

23 R. Malik, D. Burch, M. Bazant and G. Ceder, Particle size dependence of the ionic diffusivity, Nano Lett., 2010, 10, 4123-4127.

24 S. G. Kwon and T. Hyeon, Formation mechanisms of uniform nanocrystals via hot-injection and heat-up methods, Small, 2011, 7, 2685-2702.

$25 \mathrm{~W}$. Ostwald, Studien über die Bildung und Umwandlung fester Körper, Z. Phys. Chem., 1897, 22, 289-330.

26 L. Lutterotti, D. Chateigner, S. Ferrari and J. Ricote, Texture, residual stress and structural analysis of thin films using a combined X-ray analysis, Thin Solid Films, 2004, 450, 3441. 